\title{
CARACTERÍSTICAS MORFOFISIOLÓGICAS FOLIARES E ESTADO NUTRICIONAL DE SEIS ESPÉCIES LENHOSAS EM FUNÇÃO DA DISPONIBILIDADE DE ÁGUA NO SOLO
}

\author{
MORPHOPHYSIOLOGICAL LEAF CHARACTERISTICSAND NUTRITIONAL STATUS OF \\ SIX WOODY SPECIES AS A FUNCTION OF THE SOIL WATER AVAILABILITY
}

\begin{abstract}
David de Holanda Campelo ${ }^{1}$ Claudivan Feitosa de Lacerda ${ }^{2}$ João Alencar de Sousa ${ }^{3}$ Antônio Marcos

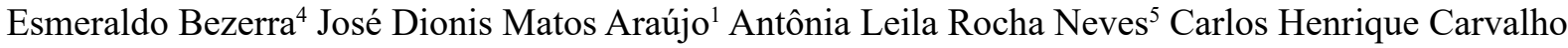
Sousa ${ }^{5}$
\end{abstract}

\section{RESUMO}

A redução da disponibilidade hídrica causa efeitos na fisiologia e morfologia das plantas, sendo responsável por alterações no metabolismo, com implicações no desenvolvimento de plantas arbóreas. O objetivo deste trabalho foi avaliar a influência do estresse hídrico no crescimento foliar e estado nutricional em plantas adultas de gonçalo-alves (Astronium fraxinifolium), guanandi (Calophyllum brasiliense Cambess.), ipê-amarelo (Handroanthus serratifolius (Vahl.), ipê-rosa (Handroanthus impetiginosa (Mart.) Matos.), marupá (Simarouba amara Aubl.) e mogno (Swietenia macrophylla King.), cultivadas em condições de sequeiro e irrigadas, no perímetro irrigado do Baixo Acaraú, Ceará. O delineamento experimental adotado foi o de medidas repetidas no tempo, num esquema de parcelas subsubdivididas (6 $62 \times 2)$, sendo a parcela principal composta por seis espécies, a subparcela por dois regimes hídricos (irrigado e sequeiro) e a subsubparcela pelas épocas de avaliação. Foram determinadas concentrações foliares de N, P e K, além de área foliar específica (AFE), grau de suculência (GS), índice relativo de clorofila (IRC) e eficiência fotossintética no uso de $\mathrm{N}(\mathrm{A} / \mathrm{N})$ e $\mathrm{P}(\mathrm{A} / \mathrm{P})$, nas estações seca e chuvosa. Os resultados mostraram que o ipêamarelo foi a espécie mais sensível quanto à variação das concentrações de $\mathrm{N}, \mathrm{P}$ e K nas folhas em resposta à disponibilidade de água no solo. Quanto à $\mathrm{AFE}$, ipê-amarelo, ipê-rosa e mogno foram mais sensíveis às condições de déficit hídrico extremo. A restrição hídrica no solo ocasiona a diminuição da suculência foliar no gonçalo-alves e no mogno. O IRC é diretamente afetado pelo déficit hídrico e está relacionado ao maior GS e à menor AFE. Quanto a $\mathrm{A} / \mathrm{N}$ e $\mathrm{A} / \mathrm{P}$, o gonçalo-alves foi a espécie mais eficiente, não sendo afetado pelas condições de disponibilidade hídrica do solo.

Palavras-chave: estresse hídrico; morfologia foliar; nutrição mineral; assimilação de $\mathrm{CO}_{2}$.

\begin{abstract}
The reduction in water availability effects plant physiology and morphology and accounts for changes in metabolism with implications for the development of tree species. The objective of this study was to evaluate the influence of water stress on morphophysiological leaf characteristics and nutritional status of adult plant of gonçalo-alves (Astronium fraxinifolium), guanandi (Calophyllum brasiliense Cambess.),

1 Engenheiro Agrônomo, MSc., Doutorando do Programa de Pós-Graduação em Engenharia Agrícola, Universidade Federal do Ceará, Av. Mister Hull, 2965, CEP 60455-760, Fortaleza (CE), Brasil. davidcamppelo@gmail.com / dionisufc@gmail.com

2 Engenheiro Agrônomo, Dr., Professor do Departamento de Engenharia Agrícola, Universidade Federal do Ceará, Av. Mister Hull, 2965, CEP 60455-760, Fortaleza (CE), Brasil. cfeitosa@ufc.br

3 Engenheiro Agrônomo, Dr., Pesquisador da Embrapa Agroindústria Tropical, Rua Drª. Sara Mesquita, 2270, CEP 60511-110, Fortaleza (CE), Brasil. joao.alencar@embrapa.br

4 Engenheiro Agrônomo, Dr., Professor do Departamento de Fitotecnia, Universidade Federal do Ceará, Av. Mister Hull, 2965, CEP 60455-760, Fortaleza (CE), Brasil. esmeraldo@ufc.br

5 Tecnólogos em Recursos Hídricos/Irrigação, Doutores em Engenharia Agrícola/Irrigação e Drenagem, Universidade Federal do Ceará, Av. Mister Hull, 2965, CEP 60455-760, Fortaleza (CE), Brasil. leilaneves7@ hotmail.com / sousaibiapina@yahoo.com.br
\end{abstract}

Recebido para publicação em 12/07/2016 e aceito em 18/05/2017

Ci. Fl., v. 28, n. 3, jul. - set., 2018 
ipê-amarelo (Handroanthus serratifolius (Vahl.), ipê-rosa (Handroanthus impetiginosa (Mart.) Matos.), marupá (Simarouba amara Aubl.) and mogno (Swietenia macrophylla King.) cultivated under rainfed and irrigated conditions, in the irrigation perimeter of Baixo Acaraú, Ceará. The experimental design was a repeated measures, a scheme of sub-divided plots $(6 \times 2 \times 2)$, the main plot consists of six species, the subplot of two water availability (irrigated and rainfed) and the sub-subplots were the evaluation time. The following evaluations were performed during the dry and rainy seasons: foliar concentrations of $\mathrm{N}, \mathrm{P}, \mathrm{K}$ and $\mathrm{Na}$, specific leaf area (AFE), leaf succulence (GS), and relative index of chlorophyll (IRC). The results showed that ipê-amarelo was the most sensitive species in terms of variation of N, P and K concentrations in leaves in response to soil water availability. Regarding AFE, ipê-amarelo, ipê-rosa and mogno were more sensitive to extreme water deficit conditions. The water restriction in the soil causes the decrease of the leaf succulence in the gonçalo alves and mogno. The chlorophyll is directly affected by drought and is related to the higher GS and the lower AFE. As for $\mathrm{A} / \mathrm{N}$ and $\mathrm{A} / \mathrm{P}$, gonçalo-alves was more efficient species, not affected by soil water availability conditions.

Keywords: water stress; leaf morphology; mineral nutrition; $\mathrm{CO}_{2}$ assimilation.

\section{INTRODUÇÃO}

O atual cenário de mudanças climáticas tem intensificado o problema da disponibilidade hídrica em regiões áridas e semiáridas em todo mundo, causado principalmente pela redução na precipitação e aumento nas taxas de evaporação (INTERGOVERNMENTAL PANEL ON CLIMATE CHANGE, 2014). Diante dessa problemática, a pesquisa de espécies adaptadas às condições de baixa disponibilidade de água no solo tem ganho importância na literatura.

Os fatores climáticos, especialmente a distribuição da precipitação, regulam o crescimento de espécies arbóreas em florestas tropicais (BRINEN; WANEK; HIETZ, 2011). Partindo-se desse princípio, o conhecimento dos fatores que modificam o crescimento nas diferentes espécies é de suma importância para o planejamento de estratégias de manejo de espécies florestais, principalmente em regiões cujos eventos de precipitação são de difícil prognóstico, como nas regiões tropicais semiáridas ou subúmidas secas.

$\mathrm{O}$ estresse hídrico é considerado um dos fatores modificadores nos processos fisiológicos e morfológicos nas plantas pela alteração no conteúdo de água na célula e pela inibição dos diversos processos de crescimento, alterando o metabolismo das plantas (TAIZ; ZEIGER, 2013). A redução da síntese no metabolismo de proteínas causa a interrupção da divisão celular, reduzindo a velocidade do processo mitótico e, assim, a redução do processo de crescimento, particularmente crescimento em extensão.

Adaptações morfológicas, fisiológicas e na anatomia são alguns dos mecanismos desenvolvidos pelas plantas quando estão sob deficiência hídrica (LARCHER, 2006). Particularmente, nesse contexto, mudanças na área da superfície, forma e na sua estrutura interna, incluindo mudanças no status da água na folha, podem ter grandes efeitos sobre os processos fisiológicos críticos da planta (TAIZ; ZEIGER, 2013). Essas alterações podem estar relacionadas, em parte, à redução do crescimento foliar em resposta à baixa disponibilidade hídrica do solo (NASCIMENTO et al., 2011; SCALON et al., 2011).

Os efeitos do estresse hídrico no estado nutricional das plantas arbóreas, assim como as estratégias de mitigação de suas consequências adversas em florestas plantadas, têm sido alvo de diversas pesquisas no âmbito ecológico pela importância que as florestas representam e, no âmbito comercial, pelo crescente aumento do consumo mundial de madeira (KREUZWIESER; GESSLER, 2010; GONÇALVES et al., 2013). Os principais nutrientes estudados são nitrogênio, fósforo e potássio, por estarem relacionados com os processos mais importantes para o desenvolvimento das plantas e sua adaptação a ambientes adversos, tais como baixa disponibilidade hídrica (SARDANS; PEÑUELAS, 2013; VILLAR-SALVADOR; PEÑUELAS; JACOBS, 2013; CHRISTINA et al., 2015).

Além de efeitos diretos no crescimento, elementos minerais como o nitrogênio e o fósforo estão ligados ao processo de assimilação de $\mathrm{CO}_{2}$ pelas plantas. A utilização eficiente desses nutrientes é uma importante estratégia para o desenvolvimento de espécies arbóreas tropicais em ambientes com recursos limitados (HIDAKA; KITAYAMA, 2009; 2013). Nesses ambientes, as plantas podem mudar a alocação de $\mathrm{N}$ foliar para maximizar o seu desempenho fotossintético, conforme mudanças no ambiente (ONODA; HIKOSAKA; HIROSE, 2004). Desta forma, o balanço entre a assimilação de $\mathrm{CO}_{2}$ e a manutenção 
da concentração do nutriente na folha pode ser considerado uma característica positiva na adaptação de espécies a ambientes com limitação hídrica.

O presente trabalho baseia-se na premissa de que o estresse hídrico causa variações nas relações entre massa e área foliar, nos padrões nutricionais e na eficiência fotossintética do uso de $\mathrm{N}$ e $\mathrm{P}$ em espécies arbóreas. Como isso ocorre para diferentes espécies são informações que podem ser usadas para auxiliar na seleção de espécies adaptadas às condições de baixa disponibilidade de água no solo. Assim, o trabalho tem como objetivo avaliar a influência do estresse hídrico nas características morfofisiológicas das folhas e no estado nutricional de seis espécies arbóreas cultivadas em condições de sequeiro e irrigadas, no perímetro irrigado Baixo Acaraú, Ceará.

\section{MATERIAL E MÉTODOS}

O trabalho foi conduzido no período de novembro de 2012 a maio de 2013 em uma área experimental da Embrapa, localizado no perímetro irrigado do Baixo Acaraú, situada a 2,2 $\mathrm{km}$ da margem direita da CE-178, no município de Acaraú, Ceará, Brasil, com coordenadas de $3^{\circ} 27^{\prime} 06^{\prime \prime}$ de latitude sul, 4008'48" de longitude oeste e altitude média de $60 \mathrm{~m}$. O clima da região, segundo a classificação de Köppen, é o Aw (tropical chuvoso), com precipitação média anual de $900 \mathrm{~mm}$, temperatura média anual de $28,1^{\circ} \mathrm{C}$, insolação de $2.650 \mathrm{~h} / \mathrm{ano}^{-1}$, umidade relativa média anual de $70 \%$, velocidade média dos ventos de $3,0 \mathrm{~m} \mathrm{~s}^{-1}$ e evaporação média anual de 1.600 mm (BRASIL, 2012).

Os dados meteorológicos do período de 2011 a 2013 foram fornecidos por dois postos climatológicos da FUNCEME e INMET, localizados no município de Acaraú, Ceará. No ano de 2011 foi observada uma precipitação total acumulada de $1.494 \mathrm{~mm}$, concentrada nos meses de fevereiro $(225,5 \mathrm{~mm})$, março $(349,1$ $\mathrm{mm})$ e abril (358,6 mm). Para o ano de 2012, a precipitação média anual foi de $491,9 \mathrm{~mm}$, concentrada nos meses de março $(139,5 \mathrm{~mm})$ e abril $(178,4 \mathrm{~mm})$. Os dados climatológicos durante o período experimental encontram-se na Tabela 1.

TABELA 1: Totais de precipitação e valores médios mensais de temperatura do ar, umidade relativa do ar e velocidade do vento no perímetro irrigado do Baixo Acaraú, Ceará.

TABLE 1: Totals rainfall and monthly average of air temperature, air humidity and wind speed in the irrigated perimeter Baixo Acaraú, Ceará.

\begin{tabular}{ccccc}
\hline Mês/Ano & $\begin{array}{c}\text { Precipitação } \\
(\mathrm{mm})\end{array}$ & $\begin{array}{c}\text { Temperatura do ar } \\
\left({ }^{\circ} \mathrm{C}\right)\end{array}$ & $\begin{array}{c}\text { Umidade } \\
\text { relativa do ar } \\
(\%)\end{array}$ & $\begin{array}{c}\text { Velocidade do vento } \\
\left(\mathrm{m} \mathrm{s}^{-1}\right)\end{array}$ \\
\hline Nov-12 & 7,2 & 28,1 & 65,9 & 4,2 \\
Dec-12 & 8,4 & 27,4 & 70,8 & 4 \\
Jan-13 & 35,9 & 28,1 & 70,5 & 3,6 \\
Feb-13 & 46,4 & 28,1 & 72,3 & 3,6 \\
Mar-13 & 79,7 & 27,9 & 73,9 & 3,5 \\
Apr-13 & 234 & 26,8 & 80,2 & 2,5 \\
May-13 & 166 & 26,9 & 78,3 & 2,9 \\
\hline
\end{tabular}

O solo da área experimental foi classificado como neossolo quartzarênico (EMBRAPA, 2013). Foram coletadas amostras de solo nas profundidades de 0-20, 20-50 e 50-80 $\mathrm{cm}$ para caracterização química e física. Os resultados das análises químicas e físicas do solo encontram-se na Tabela 2. 
TABELA 2: Caracterização físico-química do solo da área experimental, no perímetro irrigado do Baixo Acaraú, Ceará.

TABLE 2: Physical and chemical characterization of the soil of the experimental area.

\begin{tabular}{|c|c|c|c|c|c|c|c|c|c|c|c|c|}
\hline Camada & $\mathrm{pH}$ & $\mathrm{CE}$ & MO & $\mathrm{P}$ & $\mathrm{K}^{+}$ & $\mathrm{Mg}^{+}$ & $\mathrm{Ca}^{+2}$ & $\mathrm{Na}^{+2}$ & $\mathrm{H}^{+} \mathrm{Al}^{+3}$ & CTC & SB & $\mathrm{V} \%$ \\
\hline$(\mathrm{cm})$ & Água & $\mathrm{dS} / \mathrm{m}$ & $\mathrm{g} / \mathrm{Kg}$ & $\begin{array}{l}\mathrm{mg} / \\
\mathrm{dm}^{3}\end{array}$ & \multicolumn{7}{|c|}{$\mathrm{mmolc} / \mathrm{dm}^{3}$} & $\%$ \\
\hline $0-20$ & 6,4 & 0,2 & 12,9 & 22,9 & 1,6 & 7,2 & 18,8 & 3,3 & 7,5 & 38,4 & 30,9 & 80,5 \\
\hline $20-50$ & 6,1 & 0,1 & 5,1 & 16,9 & 0,5 & 5,7 & 10,5 & 3,4 & 3,3 & 23,4 & 20,1 & 86 \\
\hline $50-80$ & 5,7 & 0,1 & 5,1 & 12,8 & 0,7 & 5,4 & 8,5 & 3,5 & 10,8 & 28,9 & 18,1 & 62,2 \\
\hline
\end{tabular}

Em que: $\mathrm{CE}=$ condutividade elétrica; $\mathrm{MO}=$ matéria orgânica; $\mathrm{P}=$ fósforo; $\mathrm{K}^{+}=$potássio; $\mathrm{Mg}^{+}=$magnésio; $\mathrm{Ca}^{+2}=$ cálcio; $\mathrm{Na}^{+2}=$ sódio; $\mathrm{H}^{+} \mathrm{Al}^{+3}=$ hidrogênio e alumínio; $\mathrm{CTC}=$ capacidade de troca de cátions; $\mathrm{SB}=$ soma de bases de porcentagem de saturação por bases.

As espécies utilizadas foram gonçalo-alves (Astronium fraxinifolium Schott), guanandi (Calophyllum brasiliense Cambess.), ipê-amarelo (Handroanthus serratifolius (Vahl.), ipê-rosa (Handroanthus impetiginosa (Mart.) Matos.), marupá (Simarouba amara Aubl.) e mogno (Swietenia macrophylla King), por apresentarem características potenciais para produção de madeira e reflorestamento.

O delineamento experimental adotado foi o de medidas repetidas no tempo, em um esquema de parcelas subsubdivididas $(6 \times 2 \times 2)$, sendo a parcela principal composta por seis espécies, a subparcela por dois regimes hídricos (irrigado e sequeiro) e a subsubparcela pelas épocas de avaliação. Foram realizadas observações nas épocas seca e chuvosa na mesma planta e selecionadas quatro plantas por tratamento, considerando-se cada planta como uma repetição. Cada subparcela experimental tinha $6 \times 28 \mathrm{~m}$, constituída por três linhas de 15 plantas, sendo as linhas externas consideradas bordaduras, bem como as duas plantas dos extremos da linha central, totalizando o número de 13 plantas na área útil.

As espécies foram plantadas em outubro de 2010, sendo que, no primeiro ano, toda a área experimental foi irrigada (com uma lâmina diária aplicada de $2,7 \mathrm{~mm} \mathrm{dia}^{-1}$ ). Após esse período, as parcelas foram divididas em duas subáreas, uma que foi irrigada continuamente (regime irrigado) e outra cuja irrigação foi suspensa no fim do primeiro ano de cultivo (regime não irrigado). Foi utilizado um sistema de irrigação por microaspersão, sendo aplicada uma lâmina de $5,0 \mathrm{~mm} \mathrm{dia}^{-1}$, o que corresponde a $301 \mathrm{dia}^{-1}$ por planta, na área que permaneceu irrigada a partir do segundo ano de cultivo.

$\mathrm{O}$ espaçamento utilizado foi de $3 \mathrm{~m}$ entre as linhas e $2 \mathrm{~m}$ entre plantas. As covas de dimensões $20 \mathrm{x}$ 20 x $20 \mathrm{~cm}$ receberam adubação de base de $150 \mathrm{~g}$, composta por $120 \mathrm{~g}$ de NPK 10-28-20 mais $30 \mathrm{~g}$ de FTE BR 12 misturada ao solo no fundo da cova. Durante o período experimental, a intervalos de seis meses, cada planta recebeu adubação de cobertura por meio da incorporação de $50 \mathrm{~g}$ de NPK 10-28-20 disposto em meio círculo numa profundidade de 5 a $10 \mathrm{~cm}$.

As avaliações foram iniciadas dois anos após as mudas serem levadas ao campo, sendo as coletas de dados realizadas em 22/11/2012 (estação seca) e 17/05/2013 (estação chuvosa). Primeiramente, foram realizadas medições das trocas gasosas foliares e do teor relativo de clorofila em folhas recém-maduras e em bom estado fitossanitário, no terço médio a partir do ápice do ramo, situado na altura mediana das plantas. As leituras das trocas gasosas foliares foram realizadas com um analisador de gás infravermelho (IRGA Modelo LI-6400XT, Licor, EUA), sob luz saturante $\left(1400 \mu \mathrm{mol} \mathrm{m}^{-2} \mathrm{~s}^{-1}\right)$ e sob condições ambientais de temperatura e concentração de $\mathrm{CO}_{2}$. Em seguida, realizaram-se, nas mesmas folhas, leituras como clorofilômetro portátil Soil-Plant Analysis Development - SPAD-502 (Minolta Corp., Japão) para determinação do índice relativo de clorofila (IRC)

Foram então coletadas 15 folhas por planta amostrada, obtendo-se a massa fresca e colocando-se em sacos plásticos, acondicionados em caixas térmicas com gelo e transportados para laboratório para posterior determinação da área foliar utilizando o equipamento LI - 3100 (Li - Cor. Inc. Lincoln, Nebraska, EUA). Em seguida, todo o material foi colocado em sacos de papel, secos em estufa de circulação de forçada de ar a $65^{\circ} \mathrm{C}$ até atingir massa constante para determinações da massa seca de folhas, mensurado 
em balança analítica (BENINCASA, 2003).

De acordo com os dados de área foliar, massa seca foliar e massa fresca foliar foram calculados a área foliar específica (AFE, $\mathrm{dm}^{2} \mathrm{~g}^{-1}$ ) e o grau de suculência (GS, $\mathrm{g} \mathrm{dm}^{-2}$ ) mediante as seguintes equações, respectivamente (MANTOVANI, 1999; BENINCASA, 2003):

$\mathrm{AFE}=\frac{\mathrm{AF}}{\mathrm{MSF}}$
$\mathrm{GS}=\frac{\mathrm{MFF}-\mathrm{MSF}}{\mathrm{AF}}$

Em que: $\mathrm{AFE}=$ área foliar específica $\left(\mathrm{dm}^{2} \mathrm{~g}-{ }^{-1}\right) ; \mathrm{GS}=$ grau de suculência foliar $\left(\mathrm{g} \mathrm{dm}^{-2}\right) ; \mathrm{AF}=$ área foliar $\left(\mathrm{dm}^{2}\right.$ $\left.\mathrm{g}^{-1}\right) ; \mathrm{MSF}=$ massa seca foliar $(\mathrm{g}$ por planta); MFF = massa fresca foliar ( $\mathrm{g}$ por planta).

Para a determinação do teor de nutrientes foram utilizadas as mesmas folhas usadas para a determinação da área foliar, cujo procedimento de coleta foi descrito anteriormente. O material vegetal foi moído em moinho tipo Willey. A solubilização das amostras de tecido vegetal foi realizada por meio de digestão nítrico-perclórica para $\mathrm{P}$ e K e por meio de digestão sulfúrica para N. O nitrogênio ( $\mathrm{N}$ total) foi determinado pelo método semimicro Kjeldahl; o fósforo por espectrometria com amarelo de vanadato; o potássio por fotometria de emissão de chamas, seguindo os métodos preconizados pela Embrapa (2009). Com os dados da taxa de assimilação de $\mathrm{CO}_{2}(A)$, obtida com o IRGA, e os teores foliares de $\mathrm{N}$ e $\mathrm{P}$, foram calculadas as eficiências fotossintéticas no uso de N (A/N) e P (A/P), conforme Hidaka e Kitayama (2013) e Lacerda et al. (2016).

A umidade do solo foi determinada pelo método gravimétrico, conforme Donagema et al. (2011), nas mesmas datas de coleta de folhas. Em cada coleta foram abertas trincheiras de $60 \mathrm{~cm}$ de profundidade na linha central de cada subparcela, sendo as amostras coletadas em três profundidades $(0-20 \mathrm{~cm} ; 20-40 \mathrm{~cm}$; 40-60 cm), com três repetições em cada profundidade. Os valores de umidade na camada superficial foram de $1,3 \%$ e $4,4 \%$ nos solos cultivados sem uso da irrigação nos meses de novembro e maio, respectivamente, apresentando valores levemente maiores nas camadas mais profundas, com 2,34\% e 5,31\% correspondentes à faixa de 40-60 cm. Esses valores para as parcelas continuamente irrigadas foram 6,6\% e 5,0\% na camada de $0-20 \mathrm{~cm}$, respectivamente, para os meses de novembro e maio. Para uma análise detalhada dos perfis de umidade na área de estudo consultar Campelo et al. (2015).

Os dados obtidos foram submetidos à análise de variância com observações repetidas no tempo. Para os efeitos significativos de fatores isolados e interações foi aplicado o teste Tukey no nível de 5\% de significância, utilizando-se o software estatístico SISVAR (FERREIRA, 2008). Foi aplicado o teste de correlação de Pearson para avaliar o nível de correlação entre as variáveis pelo software SPSS versão 20.0.

\section{RESULTADOS E DISCUSSÃO}

\section{Área foliar específica, grau de suculência e índice relativo de clorofila}

Na Figura 1 encontram-se os valores de área foliar específica (AFE), grau de suculência (GS) e índice relativo de clorofila (IRC) para as seis espécies nas duas condições de regime hídrico nas épocas seca e chuvosa. Verificou-se que, na época seca, as espécies mogno, ipê-amarelo e ipê-rosa apresentaram menor AFE sob condições de sequeiro em relação às plantas da mesma espécie sob irrigação. Na época chuvosa, com o aumento da disponibilidade de água no solo, constatou-se que houve um aumento da AFE nessas espécies, exceto para o mogno. De modo geral, observou-se que a disponibilidade hídrica provida pela precipitação foi suficiente para igualar a AFE entre as plantas de sequeiro e as irrigadas em todas as espécies. Segundo Larcher (2006), folhas que se desenvolvem sob condições de suprimento hídrico deficiente são geralmente menores e apresentam menor área foliar específica.

Os resultados obtidos para as espécies do gênero Handroanthus (ipê-amarelo e ipê-rosa) são relatados na literatura (CABRAL; BARBOSA; SIMABUKURO, 2004). Nesse estudo, os autores verificaram redução 
da área foliar total em Tabebuia aurea quando submetida ao deficit hídrico. Scalon et al. (2011) constataram redução na razão de área foliar em Guazuma ulmifolia Lam. quando reduzida a disponibilidade hídrica no solo. Porém, resultados encontrados por Nascimento et al. (2011) mostraram que não houve diferenças significativas na AFE de Hymenaea courbaril L. quando submetida a diferentes conteúdos de água no solo, embora a área foliar total tenha sido afetada.

Para o grau de suculência foliar (GS) observou-se que apenas o gonçalo-alves e o mogno apresentaram significativamente menores valores de GS sob estresse hídrico, no período seco. As demais espécies não reduziram significativamente o GS quando sujeitas à baixa disponibilidade hídrica. No período chuvoso, a suculência foliar foi maior sob condições irrigadas no ipê-rosa. Os maiores valores de GS, independentemente da época de avaliação, foram observados no guanandi. $O$ fato de as espécies manterem a suculência foliar quando submetidas ao estresse hídrico pode ser visto como uma estratégia de manter a hidratação na folha por meio da estocagem de água, protegendo a planta de um murchamento repentino e de uma contração celular. Segundo Larcher (2006), uma forma especial de conservação de água é a utilização de carboidratos capazes de se hidratar (mucilagem) em células, em dutos e em cavidades entre as células. Neste trabalho, essa estratégia não foi observada no mogno e no gonçalo-alves, fato que pode estar associado às características das folhas dessas espécies, que são mais finas (maior AFE) em relação a outras espécies, com valores médios de $0,97 \mathrm{dm}^{2} \mathrm{~g}^{-1} \mathrm{e} 1,07 \mathrm{dm}^{2} \mathrm{~g}^{-1}$, característica que diminui a capacidade de estocar água nos períodos mais secos e, assim, sofrem maiores variações na perda de água nas folhas. Entretanto, essa relação não se observa para o ipê-amarelo, que apresenta alta AFE. Para Vendramini et al. (2002), a relação entre a AFE e o conteúdo de água na folha varia conforme a estratégia ecológica apresentada pelas diferentes espécies em ambientes com restrição hídrica.

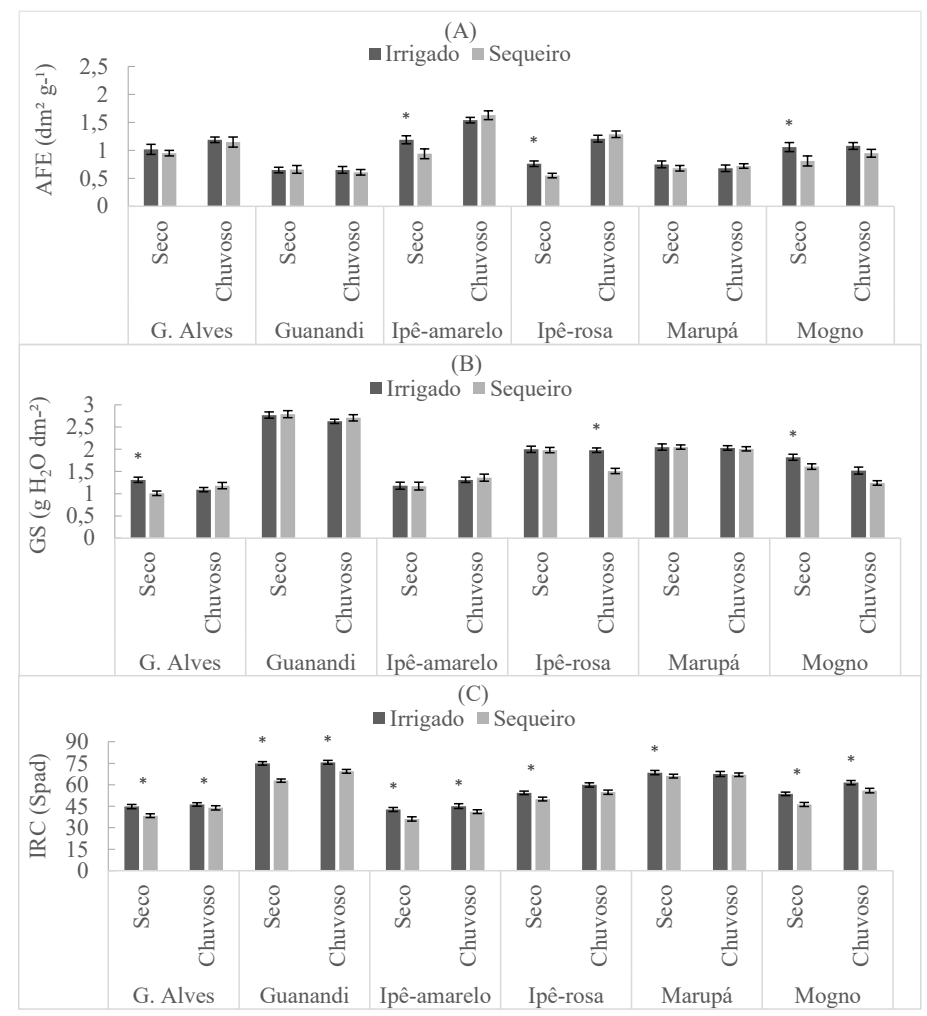

Em que: * = significativo pelo teste Tukey a 5\%

FIGURA 1: Área foliar específica - AFE (A), grau de suculência - GS (B) e índice relativo de clorofila - IRC (C) para as seis espécies submetidas a condições irrigadas e de sequeiro nas épocas seca e chuvosa. As barras de erro representam o erro padrão da média $(n=4)$.

FIGURE 1: $\quad$ Specific leaf area - AFE (A), leaf succulence - GS (B) and relative index of chlorophyll - IRC(C) for six species under irrigated and rainfed conditions in the dry and rainy season. Error bars represent standard error of the mean $(n=4)$. 
Verificou-se que o índice relativo de clorofila (IRC) foi significativamente influenciado pelo regime hídrico em todas as espécies na estação seca, com menores valores nas plantas não irrigadas. A recuperação no conteúdo de clorofila na folha na estação chuvosa foi observada apenas no marupá e no ipê-rosa. Nas demais espécies, o IRC permaneceu significativamente menor em plantas sob regime de sequeiro. Os resultados mostram que o marupá e o ipê-rosa, cultivados em sequeiro, são capazes de restabelecer os teores de clorofila na folha em resposta à maior disponibilidade hídrica. Segundo Smirnoff (1993), o estresse hídrico, em alguns níveis, favorece a formação de espécies reativas de oxigênio, que danificam, dentre outras estruturas celulares, os pigmentos fotossintéticos, o que pode explicar a redução dos teores de clorofila nessas condições. Neste trabalho, com exceção do ipê-rosa e do marupá, no período chuvoso, as demais espécies apresentaram diferença significativa em favor da condição irrigada, com os valores mais elevados sendo apresentados pelo guanandi.

A redução do conteúdo de clorofila na folha em plantas submetidas ao estresse hídrico foi observada por Khaleghi et al. (2012) em Olea europaea, quando irrigada com $40 \%$ da demanda evapotranspirativa da cultura, sofrendo redução de $46,86 \%$ na clorofila total. A redução dos pigmentos clorofilados em épocas de menor disponibilidade hídrica foi constatada em Cordia oncocalyx em mata nativa da caatinga, 50 dias após o início da seca por Mendes et al. (2013) e em seis espécies do cerrado no fim da época seca por Carvalho et al. (2007), com redução de $29 \%$ e $18 \%$ para as clorofilas $a$ e $b$, respectivamente.

Quanto à relação entre valores de IRC, AFE e GS foi encontrada correlação positiva entre o IRC e GS $\left(\mathrm{r}=0,834^{* *}\right)$ e negativa para AFE $\left(\mathrm{r}=-0,582^{* *}\right)$ (Tabela 3). Esses resultados estão de acordo com os encontrados para espécies arbóreas na Amazônia (MARENCO; ANTEZANA-VERA; NASCIMENTO, 2009). Segundo os mesmos autores, as medidas de espessura, suculência, além de massa seca foliar, interferem nas medidas de IRC realizadas por meio do SPAD.

\section{Teor de nutrientes na folha}

Na Figura 2 encontram-se os teores foliares de N, P e K para as seis espécies nas duas condições de regime hídrico nas épocas seca e chuvosa. $\mathrm{O}$ teor de $\mathrm{N}$ foi significativamente menor em plantas de ipê-amarelo e mogno cultivadas em regime de sequeiro nas duas épocas de avaliação. Nessas espécies constatou-se que o estresse hídrico limitou a absorção de N. As demais espécies não alteraram de forma significativa o conteúdo de $\mathrm{N}$ na folha, o que se pode considerar uma característica positiva para tolerar as condições de restrição hídrica. Damatta et al. (2002), estudando a interação entre disponibilidade hídrica e nutricional, verificaram o efeito positivo do nitrogênio no conteúdo relativo de água nas folhas em plantas de Coffea canephora sob restrição hídrica. A adubação nitrogenada confere maior resistência à seca por meio do aumento da sensibilidade estomática, possibilitando maior fechamento dos estômatos em condições de potencial hídrico elevado (VILLAR-SALVADOR; PEÑUELAS; JACOBS, 2013). Segundo Maathuis (2009), o nitrogênio na forma de $\mathrm{NO}_{3}{ }^{-}$é depositado no vacúolo, contribuindo de forma significativa para a turgescência celular.

Para os teores de P verificou-se que apenas o ipê-amarelo teve o teor de P nas folhas reduzido significativamente em condições de sequeiro. As demais espécies não alteraram o conteúdo foliar de $P$, independentemente do regime hídrico. A relação entre o teor de $\mathrm{P}$ e a tolerância ao estresse hídrico é pouco reportada na literatura, sendo que a maioria dos trabalhos aborda estratégias de mitigação dos seus efeitos. Contudo, a importância para a temática reside no fato da estreita relação com o nitrogênio. Sardans e Peñuelas (2013), em estudo com espécies de coníferas, sempre-verdes e decíduas na Espanha, verificaram que as maiores taxas de crescimento e biomassa acima do solo estão relacionadas com menores valores da razão N/P nas folhas. Neste trabalho, a tendência encontrada entre a relação N/P e área foliar específica foi negativa $\left(r=-0,484^{* *}\right)$ e, para o grau de suculência, positiva $\left(r=0,555^{*}\right)$, indicando que menores valores da razão N/P estão relacionadas com maior crescimento foliar, enquanto maiores valores de N/P estão relacionados com o aumento da hidratação das folhas, levando em consideração a suculência foliar (Tabela 3). Segundo Valadares et al. (2015), as características de plasticidade fenotípica de cada espécie interferem na resposta do crescimento de plantas arbóreas, principalmente na alocação de carbono para a parte aérea, em resposta ao fornecimento de fósforo.

Em relação aos teores de $\mathrm{K}$ nas folhas observou-se que gonçalo-alves, ipê-amarelo e mogno

Ci. Fl., v. 28, n. 3, jul. - set., 2018 
tiveram menores concentrações de $\mathrm{K}$ nas folhas em regime de sequeiro, na época seca. No período chuvoso houve recuperação do gonçalo-alves, guanandi, ipê-rosa e marupá. Essas espécies não alteraram de forma significativa os teores de $\mathrm{K}$ em resposta à baixa disponibilidade de água no solo, demonstrando maior capacidade na manutenção desse nutriente nas folhas sob tais condições. O potássio desempenha papel importante no estado hídrico das plantas, sendo determinante para a manutenção da turgescência celular, desempenhando importante papel na homeostase da água na planta (TAIZ; ZEIGER, 2013).

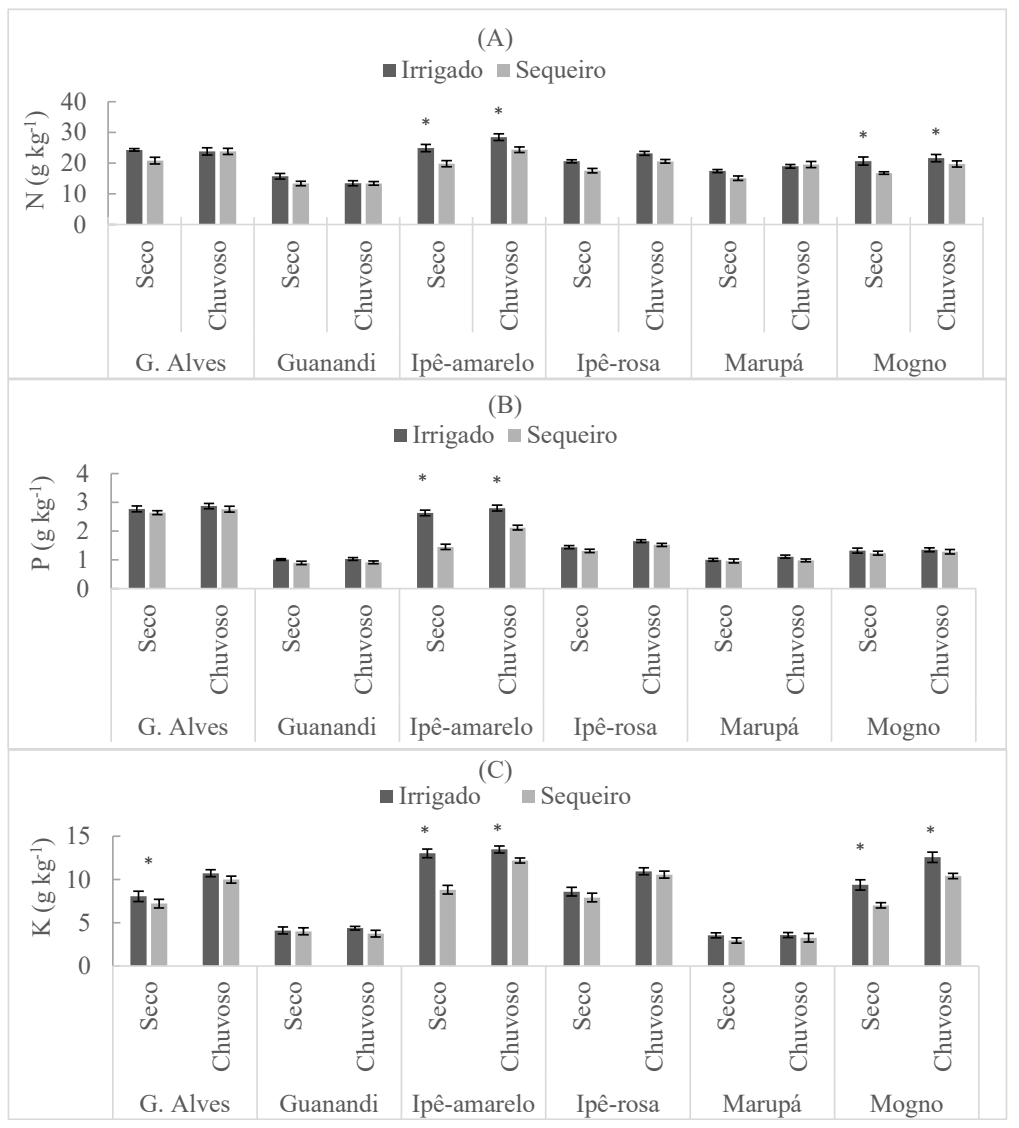

Em que: * = significativo pelo teste Tukey a 5\%

FIGURA 2: Teor foliar de Nitrogênio (A), Fósforo (B) e Potássio (C) para as seis espécies submetidas a condições irrigadas e de sequeiro nas épocas seca e chuvosa. As barras de erro representam o erro padrão da média $(n=4)$.

FIGURE 2: Leaf concetration of nitrogen (A), phosphorus (B) and potassium (C) for the six species under irrigated and rainfed conditions in the dry and rainy season. Error bars represent standard error of the mean $(n=4)$.

A regulação da abertura estomática por meio da absorção e liberação de $\mathrm{K}^{+}$está entre os principais mecanismos de controle da perda de água pelas plantas (MAATHUIS, 2009). Há diversas evidências dos efeitos benéficos do K no status de água nas plantas. Segundo Battie-Laclau et al. (2013), o acúmulo de potássio nas folhas está associado ao aumento da longevidade da folha e da área foliar, além de contribuir para o aumento da turgescência foliar por meio do acúmulo de solutos na folha. Os resultados deste trabalho estão de acordo com esses resultados, com correlação positiva entre a concentração de $\mathrm{K}$ e a área foliar específica $\left(\mathrm{r}=0,807^{* *}\right)$, porém, foram encontradas correlações negativas entre os teores de $\mathrm{K}$ e índice relativo de clorofila (IRC) $\left(\mathrm{r}=-0,674^{* *}\right)$ e grau de suculência foliar $\left(\mathrm{r}=-0,710^{* *}\right)$ (Tabela 3). As características individuais de conteúdo de clorofila em cada espécie dificultam o estabelecimento de uma relação com o conteúdo de $\mathrm{K}$, de maneira geral, assim, uma análise individual é mais indicada para obter correlação mais confiável.

A relação da concentração de $\mathrm{K}$ nas folhas e a resistência à seca em plantas arbóreas ainda é um 
tema pouco esclarecido na literatura. Estudos da interação de disponibilidade hídrica e aplicação de K e $\mathrm{Na}$ em Eucalyptus grandis mostram que não há relação entre o aumento na eficiência de uso da água em plantas e o maior fornecimento de K (EPRON et al., 2012; FREITAS et al., 2015). Entretanto, em um estudo abrangente sobre o tema, Battie-Laclau et al. (2014) concluíram que os efeitos benéficos na oferta de K sobre ajustes estruturais e fisiológicos em plantas sob estresse hídrico, tais como aumento da turgescência de células, pode não ser suficiente para contrabalancear elevados valores de índice de área foliar e condutância estomática, que também são favorecidos pelo fornecimento de K e aumentam a demanda hídrica da planta em eventos de seca extrema.

TABELA 3: Correlação de Pearson entre os teores de nutrientes na folha, área foliar específica, grau de suculência e índice relativo de clorofila.

TABLE 3: Pearson's correlation between nutrient concentrations in leaves, specific leaf area, leaf succulence and relative index of chlorophyll.

\begin{tabular}{c|ccccc}
\hline \multicolumn{1}{l}{} & AFE & GS & IRC & K & N/P \\
\hline AFE & 1 & $-0,678^{* *}$ & $-0,582^{* *}$ & $0,807^{* *}$ & $-0,484^{* *}$ \\
GS & & 1 & $0,834^{* *}$ & $-0,710^{* *}$ & $0,555^{*}$ \\
IRC & & & 1 & $-0,674^{* *}$ & $0,661^{* *}$ \\
K & & & & 1 & $-0,502^{* *}$ \\
N/P & & & & & 1 \\
\hline
\end{tabular}

Em que: $\mathrm{AFE}=$ área foliar específica; $\mathrm{GS}=$ grau de suculência; $\mathrm{IRC}=$ índice relativo de clorofila; $\mathrm{K}=$ potássio; $\mathrm{N}=$ nitrogênio; $\mathrm{P}=$ fósforo; ** = correlação significativa em nível de 0,$05 ; *$ = correlação significativa em nível de 0,01 .

\section{Eficiência fotossintética do uso de nitrogênio e fósforo}

Em relação à eficiência fotossintética do uso de nitrogênio $(\mathrm{A} / \mathrm{N})$ observa-se na Figura 3-A que, com exceção do gonçalo-alves, todas as espécies em condições de sequeiro tiveram a $\mathrm{A} / \mathrm{N}$ reduzida na época seca, em comparação com as irrigadas. Os resultados mostram que gonçalo-alves faz melhor uso do nitrogênio para a atividade fotossintética, mesmo com baixa disponibilidade de água no solo. Quanto às demais espécies, sob tais condições, verificou-se a redução da taxa de assimilação de $\mathrm{CO}_{2}$ para cada unidade de nitrogênio mineral contido nas folhas em relação ao ambiente irrigado. No período chuvoso constatou-se o aumento da $\mathrm{A} / \mathrm{N}$ em todas as plantas de sequeiro, porém, o guanandi, ao contrário das demais espécies, apresentou $\mathrm{A} / \mathrm{N}$ significativamente maior em condições irrigadas. Nesse mesmo período, os aumentos mais expressivos na $\mathrm{A} / \mathrm{N}$ ocorreram nas espécies do gênero Handroanthus (ipê-amarelo e ipê-rosa) em condições de sequeiro, superando plantas que receberam irrigação contínua.

Quanto à eficiência fotossintética do uso de fósforo $(\mathrm{A} / \mathrm{P})$ verificou-se que apenas o gonçalo-alves não teve a $\mathrm{A} / \mathrm{P}$ reduzida significativamente quando submetida à baixa disponibilidade hídrica (Figura 3-B). . O guanandi e o marupá em condição de sequeiro mantiveram a $\mathrm{A} / \mathrm{P}$ sempre menor em relação às plantas irrigadas, independentemente da época de avaliação. No período chuvoso, em condições de sequeiro, o ipê-amarelo e o ipê-rosa, ao contrário das demais espécies, apresentaram diferença significativamente superior para a relação A/P. Os maiores valores de A/P foram observados nas espécies de ipê, semelhante ao observado para a $\mathrm{A} / \mathrm{N}$.

$\mathrm{O}$ efeito do estresse hídrico nas relações $\mathrm{A} / \mathrm{N}$ e $\mathrm{A} / \mathrm{P}$ pode ser atribuído às estratégias de alocação e uso de nutrientes na folha quando essas são submetidas a condições de baixa disponibilidade hídrica. $\mathrm{O}$ investimento de $\mathrm{N}$ em biomassa de folhas pode reduzir a alocação desse elemento para o processo fotossintético e ocasionar uma queda de A/N (HIKOSAKA, 2004). Observou-se que a A/N e a A/P foram significativamente maiores em plantas que apresentaram maiores valores de massa em relação à área foliar (AFE) $\left(r=0,347^{* *}\right.$ e $0,340^{* *}$, respectivamente). Espécies como guanandi, com baixa AFE, tenderam a apresentar menores valores de A/N e A/P. Essa mesma relação foi encontrada para dez espécies arbóreas nativas da Amazônia central (MENDES; MARENCO; MAGALHÃES, 2013). Resultados semelhantes foram relatados por Poorter e Evans (1998), que afirmam que espécies com maior AFE apresentam melhor 
eficiência no uso de nitrogênio. Segundo Hidaka e Kitayama (2009), o aumento da massa em relação à área (menor AFE) diminui a A/N porque a maior fração de $\mathrm{N}$ é investida na parede celular e, consequentemente, a alocação para enzimas fotossintéticas é reduzida. Além disso, folhas com maior massa por área estão associadas à maior resistência difusiva de $\mathrm{CO}_{2}$.

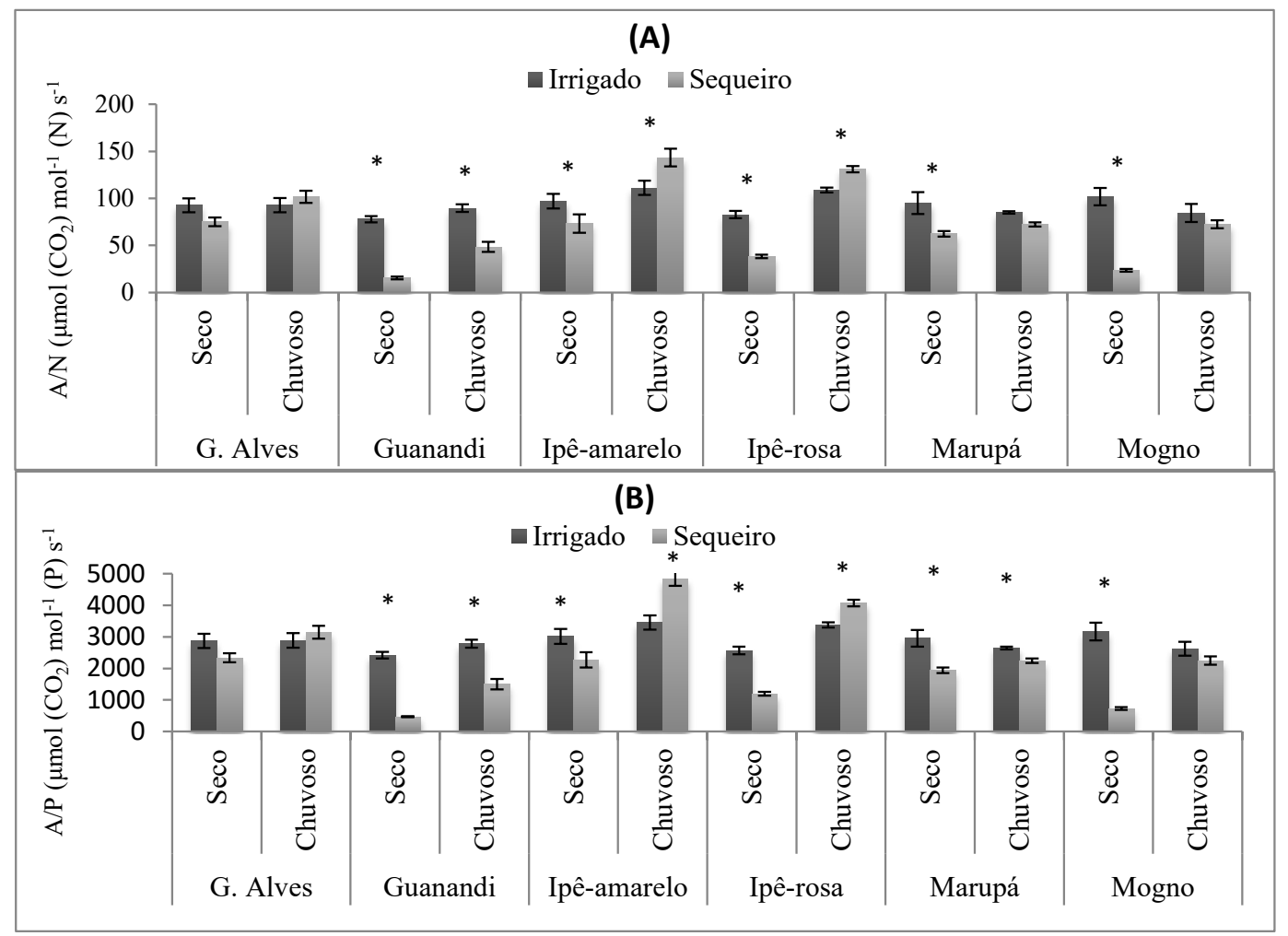

Em que: $*=$ significativo pelo teste Tukey a $5 \%$

FIGURA 3: Eficiência fotossintética do uso de nitrogênio (A) e fósforo (B) para as seis espécies submetidas a condições irrigadas e de sequeiro nas épocas seca e chuvosa. As barras de erro representam o erro padrão da média $(\mathrm{n}=4)$.

FIGURE 3: Photosynthetic nitrogen-use efficiency (A) and phosphorus (B) for the six species under irrigated and rainfed conditions in the dry and rainy season. Error bars represent standard error of the mean $(n=4)$.

O balanço entre assimilação de $\mathrm{CO}_{2}$ e o conteúdo do elemento na folha é um importante fator que deve ser levado em consideração na análise da eficiência fotossintética de nutrientes, pois ocorre de maneira diferente nas espécies. Segundo Campelo et al. (2015), para as mesmas condições deste artigo, o ipê-amarelo tem a taxa de assimilação de $\mathrm{CO}_{2}$ afetada significativamente pelo estresse hídrico, porém, as concentrações de $\mathrm{N}$ e $\mathrm{P}$ também diminuem, equilibrando o balanço de ganho de carbono e a quantidade do nutriente na folha. Por outro lado, o gonçalo-alves tem a eficiência fotossintética pouco reduzida nas mesmas condições, porém, mantém as concentrações de $\mathrm{N}$ e $\mathrm{P}$, conservando a $\mathrm{A} / \mathrm{N}$ e $\mathrm{A} / \mathrm{P}$ independentemente da disponibilidade hídrica do solo. $\mathrm{O}$ mesmo balanço não ocorre de maneira eficiente nas demais espécies estudadas. As variações interespecíficas de alocação de $\mathrm{N}$ e $\mathrm{P}$ na folha representam uma significativa adaptação das espécies para manutenção da capacidade fotossintética em resposta às mudanças ambientais. Melhorias na $\mathrm{A} / \mathrm{N}$ estão associadas ao aumento da alocação de $\mathrm{N}$ para o aparato fotossintético, principalmente para Rubisco em relação à parede celular (ONODA; HIKOSAKA; HIROSE, 2004). Com relação ao P, Hidaka e Kitayama (2013) verificaram que a A/P é fisiologicamente aumentada pela otimização da alocação e uso de $\mathrm{P}$ na folha, com o aumento no investimento de $\mathrm{P}$ em metabólitos em relação à produção de fosfolipídios. 


\section{CONCLUSÕES}

Quanto à AFE, ipê-amarelo, ipê-rosa e mogno foram mais sensíveis às condições de deficit hídrico extremo (sem irrigação no período seco).

No guanandi, ipê-amarelo e marupá, o GS não foi afetado pelas condições hídricas do solo.

Condições mais acentuadas de deficit hídrico do solo ocasionaram a diminuição da suculência foliar no gonçalo-alves e no mogno.

$\mathrm{O}$ ipê-amarelo foi a espécie mais sensível quanto à variação das concentrações de $\mathrm{N}, \mathrm{P}$ e $\mathrm{K}$ nas folhas em resposta à disponibilidade de água no solo. De maneira geral, os níveis de $\mathrm{K}$ na folha foram os mais afetados em função das condições hídricas do solo, sendo detectado em $50 \%$ das espécies estudadas.

O índice relativo de clorofila é diretamente afetado pelas condições hídricas do solo, cujo efeito foi detectado em todas as espécies estudadas e está relacionado positivamente ao GS e negativamente à AFE.

Quanto à eficiência fotossintética em relação à concentração foliar de $\mathrm{N}$ e $\mathrm{P}$, o gonçalo-alves foi a espécie mais eficiente, não sendo afetado pelas condições de disponibilidade hídrica do solo.

\section{AGRADECIMENTOS}

À CAPES pela concessão da bolsa de estudo. À Embrapa Agroindústria Tropical, à Agência de Desenvolvimento do Estado do Ceará (ADECE), ao Banco do Nordeste do Brasil (BNB) e à Universidade Federal do Ceará pelo suporte financeiro. Aos demais parceiros do projeto Embrapa Floresta, Departamento Nacional de Obras Contra as Secas (DNOCS), Sindicato das Indústrias de Móveis do Ceará (Sindmóveis), Instituto de Desenvolvimento Industrial do Ceará (INDI) e Fabricantes Associados de Marco (FAMA).

\section{REFERÊNCIAS}

BATTIE-LACLAU, P. et al. Effects of potassium and sodium supply on drought-adaptive mechanisms in Eucalyptus grandis plantations. New Phytologist, Cambridge, v. 203, n. 2, p. 401-413, jul. 2014.

BATTIE-LACLAU, P. et al. Influence of potassium and sodium nutrition on leaf area components in Eucalyptus grandis trees. Plant and soil, The Hague, v. 371, n. 1-2, p. 19-35, out. 2013.

BENINCASA, M. M. P. Análise de crescimento de plantas (noções básicas). 2. ed. Jaboticabal: FUNEP, 2003. $41 \mathrm{p}$.

BRASIL. Departamento Nacional de Obras Contra as Secas. Grupos de Coordenação Executiva das Operações Agrícolas (GOA). Situação em 30/04/1991. Fortaleza: DNOCS, 1991.

BRASIL. Departamento Nacional de Obras Contra as Secas. Perímetro irrigado baixo-acaraú. Fortaleza: DNOCS, 2012.

BRIENEN, R. J. W.; WANEK, W.; HIETZ, P. Stable carbon isotopes in tree rings indicate improved water use efficiency and drought responses of a tropical dry forest tree species. Trees: Structure and Function, Berlin, v. 25, p. 103-113, fev. 2011.

CABRAL, E. L.; BARBOSA, D. C. A.; SIMABUKURO, E. A. Crescimento de plantas jovens de Tabebuia aurea (Manso) Beth. \& Hook. f. ex S. Moore submetidas a estresse hídrico. Acta Botânica Brasileira, Manaus, v. 18, n. 2, p. 241-251, abr. 2004.

CAMPELO, D. H. et al. Trocas gasosas e eficiência do fotossistema II em plantas adultas de seis espécies florestais em função do suprimento de água no solo. Revista Árvore, Viçosa, MG, v. 39, n. 5, p. 973-983, set. 2015.

CARVALHO, A. P. F. et al. Variações sazonais nas concentrações de pigmentos e nutrientes em folhas de espécies do cerrado com diferentes estratégias fenológicas. Revista Brasileira de Botânica, São Paulo, v. 30, n. 1, p. 19-27, jan. 2007.

CHRISTINA, M. et al. Measured and modeled interactive effects of potassium deficiency and water deficit on gross primary productivity and light-use efficiency in Eucalyptus grandis plantations. Global Change Biology, Oxford, v. 21, n. 5, p. 2022-2039, maio 2015.

DAMATTA, F. M. et al. Effects of soil water deficit and nitrogen nutrition on water relations and photosynthesis of pot-grown Coffea canephora Pierre. Trees, Berlin, v. 16, n. 8, p. 555-558, nov. 2002. 
Disponível em: <http://www.dnocs.gov.br/ dnocs/doc/canais/perimetros_irrigados/ce/baixo_acarau. html>. Acesso em: 8 abr. 2013.

DONAGEMA, G. K. et al. Manual de métodos de análise de solos. 2. ed. rev. Rio de Janeiro: Embrapa Solos, 2011. $230 \mathrm{p}$.

EMBRAPA. Centro Nacional de Pesquisas de Solos. Sistema Brasileiro de Classificação de solos. 3. ed. Brasília: Embrapa Produção de Informação, Rio de Janeiro, 2013. 353 p.

EMBRAPA. Manual de análises químicas de solos, plantas e fertilizantes. 2. ed. rev. e ampl. Brasília: Embrapa Informação Tecnológica, 2009. 627 p.

EPRON, D. et al. Do changes in carbon allocation account for the growth response to potassium and sodium applications in tropical Eucalyptus plantations? Tree physiology, Oxford, v. 32, n. 6, p. 667-679, out. 2012. FERREIRA, D. F. SISVAR: um programa para análises e ensino de estatística. Revista Symposium, Lavras, v. 6, p. 36-41, jul. 2008.

FREITAS, P. C. et al. Effect of water availability and application of potassium and sodium in the juvenile wood anatomical characteristics of Eucalyptus grandis. Revista Árvore, Viçosa, MG, v. 39, n. 2, p. 405-416, mar. 2015.

GONÇALVES, J. L. M. et al. Integrating genetic and silvicultural strategies to minimize abiotic and biotic constraints in Brazilian eucalypt plantations. Forest ecology and management, Amsterdam, v. 301, p. 6-27, ago. 2013.

HIDAKA, A.; KITAYAMA, K. Divergent patterns of photosynthetic phosphorus-use efficiency versus nitrogen-use efficiency of tree leaves along nutrient-availability gradients. Journal of Ecology, Oxford, v. 97, n. 5, p. 984-991, set. 2009.

HIDAKA, A.; KITAYAMA, K. Relationship between photosynthetic phosphorus-use efficiency and foliar phosphorus fractions in tropical tree species. Ecology and evolution, [s. 1.], v. 3, n. 15, p. 4872-4880, dez. 2013.

HIKOSAKA, K. Interspecific difference in the photosynthesis-nitrogen relationship: patterns, physiological causes, and ecological importance. Journal of plant research, Tokyo, v. 117, n. 6, p. 481-494, dez. 2004. INTERGOVERNMENTAL PANEL ON CLIMATE CHANGE. IPCC 2014: climate change 2014: synthesis report. Contribution of Working Groups I, II and III to the Fifth Assessment Report of the Intergovernmental Panel on Climate Change. Geneva: IPCC, 2014. 151 p.

KHALEGHI, E. et al. Evaluation of chlorophyll content and chlorophyll fluorescence parameters and relationships between chlorophyll a, b and chlorophyll content index under water stress in Olea europaea cv. Dezful. World Academy of Science, Engineering and Technology, [s. 1.], v. 68, p. 1154-1157, ago. 2012.

KREUZWIESER, J.; GESSLER, A. Global climate change and tree nutrition: influence of water availability. Tree Physiology, Oxford, v. 30, n. 9, p. 1221-1234, set. 2010.

LACERDA, C. F. et al. Evapotranspiration as a criterion to estimate nitrogen requirement of maize under salt stress. Journal of Agronomy and Crop Science, [s. 1.], v. 202, n. 3, p. 192-202, 2016.

LARCHER, W. Ecofisiologia vegetal. São Carlos: RIMA, 2006. 531 p.

MAATHUIS, F. J. Physiological functions of mineral macronutrients. Current Opinion in Plant Biology, [s. 1.], v. 12, n. 3, p. 250-258, jun. 2009.

MANTOVANI, A. A method to improve leaf succulence quantification. Brazilian Archives of Biology and Technology, Curitiba, v. 42, n. 1, p. 1-6, 1999.

MARENCO, R. A.; ANTEZANA-VERA, S. A.; NASCIMENTO, H. C. S. Relationship between specific leaf area, leaf thickness, leaf water content and SPAD-502 readings in six Amazonian tree species. Photosynthetica, Prague, v. 47, n. 2, p. 184-190, jun. 2009.

MENDES, M. M. S. et al. Ecophysiology of deciduous plants grown at different densities in the semiarid region of Brazil. Theoretical and Experimental Plant Physiology, Campo dos Goytacazes, v. 25, n. 2, p. 97-108, ago. 2013.

MENDES, K. R.; MARENCO, R. A.; MAGALHÃES, N. S. Crescimento e eficiência fotossintética de uso do nitrogênio e fósforo em espécies florestais da Amazônia na fase juvenil. Revista Árvore, Viçosa, MG, v. 37, n. 4, p. 707-716, jul. 2013.

NASCIMENTO, H. H. C. et al. Análise do crescimento de mudas de jatobá (hymenaea courbaril 1.) em 
diferentes níveis de água no solo. Revista Árvore, Viçosa, MG, v. 35, n. 3, p. 617-626, maio 2011.

ONODA, Y.; HIKOSAKA, K.; HIROSE, T. Allocation of nitrogen to cell walls decreases photosynthetic nitrogen-use efficiency. Functional Ecology, Oxford, v. 18, n. 3, p. 419-425, jun. 2004.

POORTER, H.; EVANS, J. R. Photosynthetic nitrogen-use efficiency of species that differ inherently in specific leaf area. Oecologia, Berlin, v. 116, n. 1, p. 26-37, ago. 1998.

SARDANS, J.; PEÑUELAS, J. Tree growth changes with climate and forest type are associated with relative allocation of nutrients, especially phosphorus, to leaves and wood. Global Ecology and Biogeography, Oxford, v. 22, n. 4, p. 494-507, abr. 2013.

SCALON, S. P. Q. et al. Estresse hídrico no metabolismo e crescimento inicial de mudas de Mutambo (Guazuma ulmifolia Lam.). Ciência Florestal, Santa Maria, v. 21, n. 4, p. 655-662, out. 2011.

SMIRNOFF, N. The role of active oxygen in the response of plants to water deficit and desiccation. New Phytologist, Cambridge, v. 125, n. 1, p. 27-58, set. 1993.

TAIZ, L.; ZEIGER, E. Fisiologia vegetal. 5. ed. Porto Alegre: Artmed, 2013. 918 p.

VALADARES, S. V. et al. Plasticidade fenotípica e frações fosfatadas em espécies florestais como resposta à aplicação de fósforo. Revista Árvore, Viçosa, MG, v. 39, n. 2, p. 225-232, mar. 2015.

VENDRAMINI, F. et al. Leaf traits as indicators of resource-use strategy in floras with succulent species. New Phytologist, Cambridge, v. 154, n. 1, p. 147-157, abr. 2002.

VILLAR-SALVADOR, P.; PEÑUELAS, J. L.; JACOBS, D. F. Nitrogen nutrition and drought hardening exert opposite effects on the stress tolerance of Pinus pinea L. seedlings. Tree physiology, Oxford, v. 33, n. 2, p. 221-232, fev. 2013. 